\title{
POLYDISCS AND NONTANGENTIAL LIMITS
}

\author{
KOHUR GOWRISANKARAN
}

(Communicated by Clifford J. Earle, Jr.)

\begin{abstract}
A well-known result states that for all bounded $n$-harmonic functions on the polydisc $\mathbb{D}^{n}$ the nontangential limits exist for (Lebesgue) almost every element of the $n$-torus. In this paper it is shown that a similar result is not in general valid for bounded quotients of two positive $n$-harmonic functions. Necessary and sufficient conditions on a $n$-harmonic function $u>0$ are given to ensure the existence "almost everywhere" of the nontangential limits of the quotients $w / u$ in the case (i) for all $n$-harmonic functions $w$ such that $w / u$ is bounded and in the case (ii) for all $n$-harmonic functions $w$ that are ' $u$-quasi-bounded.'
\end{abstract}

Let $\mathbb{D}^{n}$ be the $n$-dimensional polydisc and $w$ an $n$-harmonic function on $\mathbb{D}^{n}$, i.e., a continuous real-valued function that is harmonic separately in each variable. The following is a well-known result concerning a class of those functions [8]. Suppose $w$ is $n$-harmonic and bounded on $\mathbb{D}^{n}$; then for Lebesgue almost every $\underline{t} \in \mathbb{T}^{n}$ as $\underline{z}=\left(z_{1}, \ldots, z_{n}\right)$ tends to $\underline{t}=\left(t_{1}, \ldots, t_{n}\right)$ where each $z_{j} \rightarrow t_{j}$ nontangentially (and to stress the condition independent of the other variables) $w(\underline{z})$ converges to a real number. In terms of potential theoretical expectations it is natural to expect the result to extend to bounded quotients of positive $n$-harmonic functions. More precisely, suppose $u>0$ is an $n$ harmonic function on $\mathbb{D}^{n}$ that is represented as the integral of a finite Borel measure $\mu_{u}$ on $\mathbb{T}^{n}$ relative to the product of the Poisson kernels $[4,8]$. Let $w$ be any $u$-bounded (i.e., $|w / u|$ bounded) $n$-harmonic function on $\mathbb{D}^{n}$. It is natural to expect the nontangential limits of $w / u$ to exist for $\mu_{u}$ almost every element of $\mathbb{T}^{n}$.

The first main result of this paper will prove this expectation to be false. We give further a necessary and sufficient condition on $u$ under which the result is valid for all $u$-bounded $n$-harmonic functions. With a slightly different perspective it is natural to ask if the nontangential limits exist almost everywhere on $\mathbb{T}^{n}$ for quasi-bounded $n$-harmonic functions. We consider the problem in the general situation of $u$-quasi-bounded $n$-harmonic functions. We recall that a positive $n$-harmonic function is said to be $u$-quasi-bounded if it is the limit

Received by the editors August 9, 1989 and, in revised form, December 27, 1990.

1980 Mathematics Subject Classification (1985 Revision). Primary 31B25; Secondary 32A40, $31 \mathrm{C} 99$.

Key words and phrases. Nontangential limit, $n$-harmonic functions, polydisc, quasi-bounded functions, fine limit.

Presented at the International Conference on Potential Theory, Nagoya, 1991. 
of an increasing sequence of positive $u$-bounded functions. We give necessary and sufficient conditions on $u$ to ensure the existence of nontangential limits of $w / u$ at $\mu_{u}$ almost every element of $\mathbb{T}^{n}$ and for all $u$-quasi-bounded $n$ harmonic functions $w$. One of the principal tools used in our proofs is the existence of iterated fine limits of quotients of $n$-harmonic functions [7]. Another development, which is probably of independent interest, is the introduction of a restructured PWB (for Perron-Wiener-Brelot) problem in our situation. We want to thank the referee for some valuable comments and, in particular, for the much simpler proof of Theorem 2 .

In what follows we assume that $u>0$ is a $n$-harmonic function on $\mathbb{D}^{n}$ and is represented uniquely by the finite Borel measure $\mu_{u}$ on $\mathbb{T}^{n}$ relative to the product of the Poisson kernels.

We now have

Lemma 1. Let $w>0$ be a $n$-harmonic function represented by the finite Borel measure $\nu_{w}$ on $\mathbb{T}^{n}$. Then the iterated nontangential limits of $w / u$ exist for $\mu_{u}$ almost every element of $\mathbb{T}^{n}$. The limit function (determined up to a set of $\mu_{u}$ measure zero) is a Radon-Nikodym derivative of the absolutely continuous part of $\nu_{w}$ relative to $\mu_{u}$.

Proof. We know that the iterated fine limits of $w / u$ exist $\mu_{u}$ almost everywhere and the limit function is the required Radon-Nikodym derivative [7]. However, by [1, Theorem 4], we conclude that at every stage the existence of the fine limit implies that of the nontangential limit, equal to the fine limit. Thus the iterated nontangential limit of $w / u$ exists $\mu_{u}$ almost everywhere and verifies the required condition. The proof is complete.

Corollary A. If in addition $w$ is u-quasi-bounded then $g d \mu_{u}$ represents the function $w$ where $g$ is the iterated limit function.

Proof. It is simple to verify that $w>0$ is $u$-quasi-bounded if and only if the measure $\nu_{w}$ on $\mathbb{T}^{n}$ representing $w$ is absolutely continuous relative to $\mu_{u}$. The corollary is then easily seen to be true.

The following corollary is an immediate consequence.

Corollary B. Suppose $w$ is as in the above corollary and assume, in addition, that the nontangential limit of $w / u$ exists for $\mu_{u}$ almost every element of $\mathbb{T}^{n}$. Then the nontangential limit function $g$ is such that $g d \mu_{u}$ is the representing measure of $w$.

Definition. Suppose $f$ is an extended real-valued function on $\mathbb{D}^{n}$. For the element $\underline{t}=\left(t_{1}, \ldots, t_{n}\right) \in \mathbb{T}^{n}$, we refer to nontangential $\lim \sup f(\underline{z})$ as $\underline{z}=$ $\left(z_{1}, \ldots, z_{n}\right) \rightarrow \underline{t}$ as the extended real number $a$ that is the supremum of the limit values of $\bar{f}(z)$ when $\underline{z} \rightarrow \underline{t}$ nontangentially as explained at the beginning. It is easily verified that $a$ is also the supremum as $\underline{\theta} \rightarrow \pi / 2$ of the $\lim \sup f(\underline{z})$ as $\underline{z} \rightarrow \underline{t}$ and $\underline{z}$ stays in the product of Stolz domains of semivertical angles $\left(\theta_{1}, \ldots, \theta_{n}\right)=\underline{\theta}[1]$.

Theorem 2. There exist n-harmonic functions $u$ and $w$ both $>0, w / u$ bounded such that the nontangential limit of $w / u$ does not exist for a set of elements of $\mathbb{T}^{n}$, of positive ( $\left.>0\right) \mu_{u}$ measure, where $\mu_{u}$ is the canonical (finite Borel) measure on $\mathbb{T}^{n}$ representing $u$. 
Proof. We recall that there exists an $n$-harmonic function $w>0$ such that the nontangential $\lim \inf w=0$ and nontangential $\lim \sup w=+\infty$ for (Lebesgue) almost every element of $\mathbb{T}^{n}[2,5]$. Let $u=w+1$ where $w$ is the function as above. Then $u>0$ is $n$-harmonic on $\mathbb{D}^{n}$ and $w$ is $u$-bounded. Further we note that the canonical measure $\mu_{u}$ is such that $\mu_{u} \geq$ the Lebesgue measure on $\mathbb{T}^{n}$. Clearly, the nontangential $\lim \inf w / u=0$ and nontangential $\lim \sup w / u=1$ for (Lebesgue) almost every element of $\mathbb{T}^{n}$. In particular, the same holds for a subset of $\mathbb{T}^{n}$ with $\mu_{u}$-measure $>0$. This proves the theorem.

Theorem 3. Let $u>0$ be a $n$-harmonic function with the representing measure $\mu_{u}$. For every Borel subset $E \subset \mathbb{T}^{n}$, let $u_{E}$ be the n-harmonic function with representing measure $\chi_{E} d \mu_{u}$. The following two conditions are equivalent.

(1) For every u-bounded $n$-harmonic function $w$, the nontangential limit of $w / u$ exists for $\mu_{u}$ almost every element of $\mathbb{T}^{n}$.

(2) For every Borel set $E \subset \mathbb{T}^{n}$, the nontangential limit of $u_{E} / u$ equals zero for $\mu_{u}$ almost every element in $\mathbb{T}^{n} \backslash E$.

Proof. Suppose that the condition (1) is verified for $u$. Then by the corollary $\mathrm{B}$, the nontangential limit of $u_{E} \backslash u$ equals $\chi_{E}, \mu_{u}$ almost everywhere on $\mathbb{T}^{n}$.

Conversely, suppose that condition (2) is verified for $u$. Let $E$ be an arbitrary Borel set. Since (2) is valid both for $E$ and $\mathbb{T}^{n} \backslash E$, we conclude easily that the nontangential limit of $u_{E} / u$ equals $\chi_{E}, \mu_{u}$ almost everywhere. Suppose $f$ is any bounded Borel function on $\mathbb{T}^{n}$ and $\sigma(f)$ the $n$-harmonic function that is the integral of the product of Poisson kernels relative to the signed measure $f d \mu$. Every $u$-bounded $n$-harmonic function is necessarily of this form. Simple measure theoretic arguments let us conclude that the nontangential limit of $\sigma(f) / u$ exists $\mu_{u}$ almost everywhere on $\mathbb{T}^{n}$. Then we conclude by Corollary B that the above limit equals $f, \mu_{u}$ almost everywhere. The proof is complete.

Let us now prove the following restricted version of a minimum principle for $n$-harmonic functions.

Theorem 4. Let $w$ be a $n$-harmonic function on $\mathbb{D}^{n}$ such that $w / u \geq-\alpha$ for some positive number $\alpha$. Let further the nontangential lim inf of $w / u$ be $\geq 0$ for $\mu_{u}$ almost every element of $\mathbb{T}^{n}$. Then $w \geq 0$.

Proof. The positive $n$-harmonic function $w+\alpha u$ can be uniquely expressed as the sum $w_{1}+w_{2}$ of positive $n$-harmonic functions where the representing measure of $w_{1}$ (resp. $w_{2}$ ) is absolutely continuous (resp. singular) relative to $\mu_{u}$. From the hypothesis of the theorem and Lemma 1 we know the iterated (fine as well as) nontangential limit of $(w / u+\alpha)$ exists and majorizes $\alpha, \mu_{u}$ almost everywhere. We further conclude that $w_{1} \geq \alpha u$.

Hence, $w+\alpha u=\left(w_{1}-\alpha u\right)+\alpha u+w_{2}$ where each term on the right is a positive $n$-harmonic function. Hence $w \geq 0$ completing the proof.

We now proceed to consider the nontangential behavior of $u$-quasi-bounded $n$-harmonic functions. Let us assume that the positive $n$-harmonic function $u$ verifies either of the equivalent hypothesis stated in Theorem 3. Let us have the convention that $\operatorname{Inf} \phi=\infty$. For an extended real-valued function $f$ defined 
on $\mathbb{T}^{n}$ we define the following lower envelope:

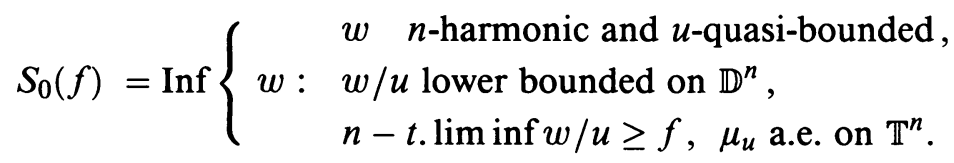

Modifying the envelope introduced by Doob [3] we define for all $f \geq 0$ on $\mathbb{T}^{n}$

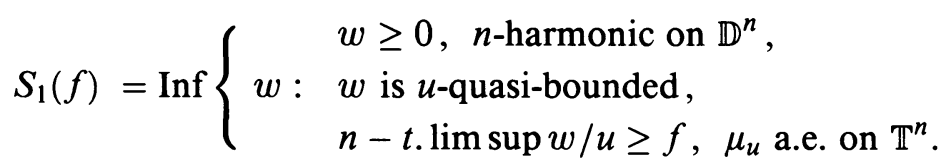

Theorem 5. $S_{0}(f)$ is identically $\pm \infty$ or it is a $n$-harmonic function on $\mathbb{D}^{n}$.

Proof. We may assume that $S_{0}(f) \not \equiv+\infty$. Suppose $w_{1}$ and $w_{2}$ are two of the functions in the class defining $S_{0}(f)$. Define for each positive integer $m$,

$$
h_{m}=\text { greatest } n \text {-harmonic minorant of } \min \left(w_{1}, w_{2}, m u\right)
$$

[4]. Clearly $h_{m}$ exists and is $u$-bounded and hence the nontangential limit of $h_{m} / u$ exists $\mu_{u}$ almost everywhere.

Now, $v_{m}=\min \left(w_{1}, w_{2}, m u\right)-h_{m}$ is a nonnegative $n$-superharmonic function with greatest $n$-harmonic minorant zero. Further the iterated nontangential as well as iterated fine limit of $v_{m} / u$ exists $\mu_{u}$ a.e. and is hence $=0$, $\mu_{u}$ a.e. It follows therefore that $n-t$.liminf $v_{m} / u=0, \mu_{u}$ a.e. Hence, the $n-t . \liminf \min \frac{1}{u}\left(w_{1}, w_{2}, m u\right)=n-t . \lim h_{m} / u, \mu_{u}$ a.e. This implies that the $n-t \cdot \lim h_{m} / u$ is $\geq \min (f, m), \mu_{u}$ a.e. Clearly the sequence of $n$ harmonic functions $h_{m}$ increases and by Harnack property [4] converges to a $n$-harmonic function $h$ as $m$ tends to $\infty$ and $h \leq \min \left(w_{1}, w_{2}\right)$. Let now $h^{\prime}$ be the greatest $n$-harmonic minorant of $\min \left(w_{1}, w_{2}\right)$. Clearly, $h^{\prime} / u$ is lower bounded and further

$$
n-t \cdot \liminf h^{\prime} / u \geq n-t \cdot \liminf h / u \geq \min (f, m)
$$

$\mu_{u}$ a.e. for each $m$. We conclude that $h^{\prime} \geq S_{0}(f)$. Now it is easy that $S_{0}(f)$ may be realized as the limit of a decreasing directed family of $n$-harmonic functions and is hence, by Harnack property, necessarily $-\infty$ identically or a $n$-harmonic function on $\mathbb{D}^{n}$. The proof is complete.

Lemma 6. $S_{0}(f)$ introduced above satisfies the following properties.

(a) $S_{0}(f)$ increases with increasing $f$.

(b) Suppose $f_{n} \uparrow f, f_{1} \geq 0$ then $S_{0}\left(f_{n}\right) \uparrow S_{0}(f)$.

(c) $S_{o}(f) \geq-S_{0}(-f)$.

Proof. The first property is trivial and the third one is an immediate consequence of Theorem 4. The proof of (b) follows the familiar pattern. Suppose $f_{n} \geq 0$ and increases to $f$. We need to consider the only case when $\lim S_{0}\left(f_{n}\right) \not \equiv+\infty$ for each $n$. Fix $\underline{z} \in \mathbb{D}^{n}$ and $\varepsilon>0$. Choose for each $n$, a $u$-quasi-bounded $n$-harmonic function $w_{n} \geq 0, w_{n}$ in the defining family of $S_{0}\left(f_{n}\right)$ such that $S_{0}\left(f_{n}\right)(\underline{z})+\varepsilon / 2^{n}>w_{n}(\underline{z})$. It is simple to verify that for each $n, S_{0}\left(f_{n}\right)$ is $u$-quasi-bounded. Clearly the limit $v$ of (the increasing sequence) $S_{0}\left(f_{n}\right)$ is $n$-harmonic and also $u$-quasi-bounded. Let us now set $w=v+\sum_{1}^{\infty}\left[w_{n}-S_{0}\left(f_{n}\right)\right]$. Clearly, $w$ is a positive $u$-quasi-bounded 
$n$-harmonic function such that nontangential liminf $w / u \geq f, \mu_{u}$ a.e. Hence $w \geq S_{0}(f)$. It follows that $S_{0}(f)(\underline{z}) \leq v(\underline{z})+\varepsilon$. The proof is completed easily.

Theorem 7. For all Borel functions $f \geq 0$

$$
S_{0}(f)=\sigma(f)=\int f \mathbb{P} d \mu_{u},
$$

where $\mathbb{P}$ is the product of the Poisson kernels.

Proof. Consider first a Borel function $f$ such that $0 \leq f \leq M$ for some M. $\sigma(f) \geq 0$ and besides by our hypothesis, $\sigma(f) / u$ has nontangential limits equal to $f, \mu_{u}$ a.e. and hence $\sigma(f) \geq S_{0}(f)$. However, it is easy to check that $-\sigma(f) \geq S_{0}(-f)$ or $\sigma(f) \leq-S_{0}(-f) \leq S_{0}(f)$. Hence, $\sigma(f)=S_{0}(f)$ in this case. In general, if $f \geq 0$ and Borel, set $f_{n}=\min (f, n)$. Then, the above lemma and monotone convergence theorem gives us

$$
\sigma(f)=\lim \sigma\left(f_{n}\right)=\lim S_{0}\left(f_{n}\right)=S_{0}(f) .
$$

The proof is complete.

Now we introduce a stronger condition to ensure the existence of nontangential limits for $u$-quasi-bounded functions.

Let $f \geq 0$ be Borel and $\mu_{u}$ integrable on $\mathbb{T}^{n}$. Then the nontangential limit of $\sigma(f) / u$ exists and equals zero for $\mu_{u}$ almost every $\underline{t} \in N_{f}=\{\underline{t}: f(t)=0\}$.

It is easy to deduce the hypothesis (2) of Theorem 3 in case $u$ satisfies the above condition $(\mathrm{H})$; hence it is stronger than the requirement of the existence of limits for $u$-bounded functions.

Theorem 8. Suppose the $n$-harmonic function $u>0$ satisfies the condition $(\mathrm{H})$ above. Then for all $\mu_{u}$ integrable function $f \geq 0$ on $\mathbb{T}^{n}, S_{0}(f)=S_{1}(f)$.

Proof. It is clear that $S_{0}(f) \geq S_{1}(f)$. We want to prove the opposite inequality. As before, let us first assume that $f$ is in addition bounded. Let $w$ be $\geq$ $0, n$-harmonic and $u$-quasi-bounded and verify nontangential limsup $w / u \geq$ $f, \mu_{u}$ a.e. Suppose $w=\sigma(g)$ for some $g \geq 0$ and Borel and let $v=$ $\min (\sigma(g), \sigma(f))$. Since the nontangential $\lim \sigma(f) / u=f, \mu_{u}$ a.e., we have that the nontangential $\lim \sup v / u \geq f, \mu_{u}$ a.e.

Now, it is easy to verify that $\sigma(\min (f, g))=h$ is the greatest $n$-harmonic minorant of $v$. Further,

$$
v-h=\min [\sigma(g-\min (f, g)), \sigma(f-\min (f, g))] .
$$

Let $E_{1}=\left\{\underline{t} \in \mathbb{T}^{n}: g(\underline{t})>f(\underline{t})\right\}$ and $E_{2}$ the complement of $E_{1}$. Clearly, for all $\underline{t} \in E_{1}, \min (f(\underline{t}), g(\underline{t}))=f(\underline{t})$ and hence, nontangential $\lim h / u=$ $\min (f(\underline{t}), g(\underline{t}))=f(\underline{t})$ except on a set of $\mu_{u}$ measure zero. However, for $\mu_{u}$ almost every $\underline{t} \in E_{2}$ that is the set where the $\mu_{u}$ integrable nonnegative function $g-\min (f, g)$ takes the value 0 , and (by the condition $H$ ), $\frac{1}{u} \sigma[g-\min (f, g)] \rightarrow 0$. Hence, the nontangential limit $(v-h) / u=0, \mu_{u}$ a.e. on $E_{2}$. In particular, we conclude that at all those $\underline{t}$, the nontangential limit of $h / u$ equals $f, \mu_{u}$ a.e. Since $h=\sigma(\min (f, g))$ and is $u$-bounded, we deduce that $\min (f, g)=f, \mu_{u}$ a.e., i.e., $\sigma(g) \geq \sigma(f)$. 
This is true for all $w=\sigma(g)$ in the defining class of $S_{1}(f)$, and we conclude that $S_{1}(f) \geq \sigma(f)=S_{0}(f)$. The required result is therefore proved for $f \geq 0$ bounded and Borel on $\mathbb{T}^{n}$. In general, for any $f \geq 0$ Borel and $\mu_{u}$-integrable, let $f_{n}=\min (f, n)$, for all $n$. Then,

$$
S_{1}(f) \geq S_{1}\left(f_{n}\right)=S_{0}\left(f_{n}\right) / S_{0}(f) \geq S_{1}(f) .
$$

The proof is complete.

We include the following result for the sake of completeness.

Theorem 9. Let $f: \mathbb{D}^{n} \rightarrow \mathbb{R}$ be a continuous function. Let $\tilde{f}(\underline{t})=$ nontangential $\lim \sup f(\underline{z})$ as $\underline{z} \rightarrow \underline{t}$, for all $\underline{t} \in \mathbb{T}^{n}$. Then, $\tilde{f}$ is a Borel function.

Proof. Let $\theta$ be a fixed number $0<\theta<\pi / 2$. Let $R_{\theta, \delta}(\underline{t})$ be the nontangential domain in $\mathbb{D}^{n}$ with vertex $\underline{t}$, which is the product of the Stolz domains $S_{j}(\theta, \delta)$ with vertex $t_{j}$, of semivertical angle $\theta$ and cut off by a circle of radius $\delta$ centered at $t_{j}$. Let $m$ be a sufficiently large number so that the closed polydisc $\mathbb{D}_{m}^{n}$ of radius $1-\frac{1}{m}$ has nonvoid intersection with $R_{\theta, \delta}$. From the fact that $f$ is uniformly continuous on $\mathbb{D}_{m}^{n}$ we deduce easily that $g_{m}$ defined by $g_{m}(\underline{t})=$ $\sup f(\underline{z})$ for $z \in R_{\theta, \delta}(\underline{t}) \cap \mathbb{D}_{m}^{n}$ is (uniformly) continuous on $T^{n}$. It is clear that if $\ell_{\theta}(\underline{t})=\lim _{\delta \rightarrow 0}\left[\lim _{m \rightarrow \infty} g_{m}(\underline{t})\right]$ then $\ell_{\theta}(\underline{t})=\limsup f(\underline{z})$ as $\underline{z} \rightarrow \underline{t}$ and $\underline{z}$ staying in the product of Stolz domain of angle $\theta$. Hence, $\ell_{\theta}$ is a Borel function; in fact, with very minimal restrictions the limit of a decreasing sequence of lower semicontinuous functions on $\mathbb{T}^{n}$. As we have remarked earlier the nontangential limsup of $f$ is the increasing limit of the functions $\ell_{\theta}$ as $\theta \uparrow \pi / 2$. The theorem is proved.

Theorem 10. Let $u>0$ be an $n$-harmonic function on $\mathbb{D}^{n}$ that verifies the condition $(\mathbf{H})$. Let $w$ be the integral of the product of Poisson kernels relative to the (signed) measure $f d \mu_{u}$ where $f$ is a $\mu_{u}$-integrable Borel function on $\mathbb{T}^{n}$. Then the nontangential limit of $w / u$ exists $\mu_{u}$ a.e. and equals $f$.

Proof. Clearly, it is enough to prove the result for $f \geq 0$. Let $\tilde{f}$ be the nontangential limsup function associated to $\sigma(f) / u$. By the last theorem, $\tilde{f}$ is a Borel function and we know that $\tilde{f} \geq n-t \liminf \sigma(f) / u \geq f, \mu_{u}$ a.e. However, by Theorems 8 and 7

$$
\sigma(f) \geq S_{1}(\tilde{f})=S_{0}(\tilde{f})=\sigma(\tilde{f}) \geq \sigma(f) .
$$

This clearly implies that $f=\check{f}, \mu_{u}$ a.e. The proof is complete.

Theorem 11. Let $u_{j}, j=1,2$ be a positive $n$-harmonic function on $\mathbb{D}^{n}$ with the canonical representing Borel measure $\mu_{j}$ on $\mathbb{T}^{n}$ such that the condition $(\mathrm{H})$ is verified by $u_{j}$. Then the following are results concerning the corresponding properties of $\left(u_{1}+u_{2}\right)$.

(a) Every $\left(u_{1}+u_{2}\right)$-bounded $n$-harmonic function $\mathbb{D}^{n}$ has a nontangential limit for $\left(\mu_{1}+\mu_{2}\right)$ almost every element of $\mathbb{T}^{n}$ iff the nontangential limit of $u_{j} / u_{k}$ exists as a real number for $\mu_{k}$ almost every element of $\mathbb{T}^{n}$, for $j, k=1,2$.

(b) Let $\nu_{j}$ be the singular part of the measure $\mu_{j}$ relative to $\mu_{k}$. Then $\left(u_{1}+\right.$ $\left.u_{2}\right)$ satisfies the property $(\mathrm{H})$ iff for all positive $\left(\mu_{1}+\mu_{2}\right)$-integrable Borel functions $f,\left(\int f \mathbb{P} d \nu_{j}\right) / u_{k}$ has nontangential limit 0 for $\mu_{k}$ almost every element of $\mathbb{T}^{n}$ for $j=1,2$ and $k \neq j$. 
Proof. (a) Let $g_{j}$ be the Radon-Nikodym derivative of the absolutely continuous part of $\mu_{j}$ relative to $\mu_{k}$. Then, $d \mu_{j}=g_{j} d \mu_{k}+d \nu_{j}$. Assume that $u_{j} / u_{k}$ has the required property. Let $E$ be any Borel set. Then

$$
\frac{\left(u_{1}+u_{2}\right)_{E}}{u_{1}+u_{2}}=\frac{1}{u_{k}}\left[\left(u_{k}\right)_{E}+\int_{E} g_{j} \mathbb{P} d \mu_{k}+\int \mathbb{P} d \nu_{j}\right] /\left[1+\frac{u_{j}}{u_{k}}\right],
$$

where $\mathbb{P}$ is the product of the Poisson kernels. For $k \neq j, 0 \leq \int_{E} \mathbb{P} d \nu_{j} / u_{k} \leq$ $\int \mathbb{P} d \nu_{j} / u_{k}$ tends to zero $\mu_{k}$-a.e. and the function $g_{j}$ being clearly Borel and $\mu_{k}$ integrable we conclude that the above quotient has a nontangential limit equal to zero $\mu_{k}$ almost everywhere in the complement of $E$. This being true for $k=1,2$ we conclude that the condition of Theorem 3 is fulfilled for $\left(u_{1}+u_{2}\right)$.

Conversely, the assumption on $\left(u_{1}+u_{2}\right)$ clearly implies that $u_{j} /\left(u_{j}+u_{k}\right)$ has nontangential limit $\left(\mu_{1}+\mu_{2}\right)$ almost everywhere for $j=1,2$. This establishes in particular that $u_{k} / u_{j}$ has nontangential limit (including possibly $\left.+\infty\right)\left(\mu_{1}+\right.$ $\mu_{2}$ ) almost everywhere. Using the fact that this limit is indeed the iterated nontangential limit we conclude by the Lemma 1 that the nontangential limit (function) of $u_{k} / u_{j}$ is the equal to $g_{k}$ ( $\mu_{j}$ almost everywhere).

Let us now prove (b). Let $g_{j}, \nu_{j}$ be as above. Suppose $\left(u_{1}+u_{2}\right)$ satisfies (H) and $f \geq 0$ and belongs to $L^{1}\left(\mu_{1}+\mu_{2}\right)$. Let $F_{j}$ be a Borel set carrying the measure $\nu_{j}$. We have,

$$
n-t . \lim \frac{\int f \mathbb{P} d \nu_{j}}{u_{k}}=n-t \cdot \lim \left(\int_{F_{j}} f \mathbb{P}\left(d \mu_{1}+d \mu_{2}\right)\right) \frac{1}{u_{k}}, \quad \mu_{k} \text { a.e. },
$$

since $\mu_{k}\left(F_{j}\right)=0$ and $\mu_{j} / F_{j}=\nu_{j}$. Clearly, by dividing the numerator and denominator of the above quotient by $\left(u_{1}+u_{2}\right)$ and taking the limit, we get that this nontangential limit function equals $\left(f \chi_{F_{j}}\right)\left(1+g_{j}\right)$. Hence it is zero $\mu_{k}$ almost everywhere. This is true for $k=1,2$.

Conversely, suppose $u_{1}$ and $u_{2}$ verify all the listed conditions and $f$ be in $L^{1}\left(\mu_{1}+\mu_{2}\right)$ and $f \geq 0$. We have

$$
\tau(f)=\int f \mathbb{P}\left(d \mu_{1}+d \mu_{2}\right)=\int f \mathbb{P} d \mu_{k}+\int f \mathbb{P} g_{j} d \mu_{k}+\int f \mathbb{P} d \nu_{j} .
$$

We can express $\tau(f) /\left(u_{1}+u_{2}\right)$ as $\left(\tau(f) / u_{k}\right)\left(u_{k} / u_{1}+u_{2}\right)$ and consider the nontangential limits of $\tau(f) / u_{k}$. It is trivial to see that nontangential limit of $u_{j} / u_{k}$ exists $\mu_{k}$ almost everywhere for $j, k=1,2$. Clearly, the first two terms in the sum tend respectively to $f$ and $f g_{j}, \mu_{k}$ almost everywhere. The last term tends to zero $\mu_{k}$ almost everywhere by our hypothesis. In particular, $\tau(f) /\left(u_{1}+u_{2}\right)$ tends to zero at all points where $f=0, \mu_{k}$ almost everywhere. This is true for $k=1,2$. Thus we have shown that $\left(u_{1}+u_{2}\right)$ satisfies the property $(\mathrm{H})$. The proof is complete.

\section{BiBLIOGRAPHY}

1. M. Brelot and J. L. Doob, Limites angulaires et limites fines, Ann. Inst. Fourier (Grenoble) 13 (1963), 395-415.

2. A. P. Calderon and A. Zygmund, Note on the boundary values of functions of several complex variables, Contributions to Fourier Analysis, Ann. of Math. Stud., no. 25, Princeton Univ. Press, Princton, NJ, 1950, pp. 145-165.

3. J. L. Doob, A non-probabilistic proof of a relative Fatou Theorem, Ann. Inst. Fourier (Grenoble) 9 (1959), 293-300. 
4. K. Gowrisankaran, Multiply harmonic functions, Nagoya J. Math. 28 (1966), 22-48. MR35 \#410

5. 39 (1970), 127-132.

6. _ Iterated fine limits and iterated non-lingential limits, Trans. Amer. Math. Soc. 173 (1972), 71-92. MR47 \#489

7. __ Iterated fine limits, Proc. Amer. Math. Soc. 108 (1990), 157-162.

8. A. Zygmund, Trigonometrical series, Vol. 2, 2nd rev. ed. Cambridge Univ. Press, New York, 1959.

Department of Mathematics \& Statistics, McGill University, 805 Sherbrooke W, Montreal H3A 2K6, Canada 